\title{
On uniqueness of automorphisms groups of Riemann surfaces
}

\author{
Maximiliano Leyton A. and Rubén A. Hidalgo
}

\begin{abstract}
Let $\gamma, r, s, \geq 1$ be non-negative integers. If $p$ is a prime sufficiently large relative to the values $\gamma, r$ and $s$, then a group $H$ of conformal automorphisms of a closed Riemann surface $S$ of order $p^{s}$ so that $S / H$ has signature $(\gamma, r)$ is the unique such subgroup in $\operatorname{Aut}(S)$. Explicit sharp lower bounds for $p$ in the case $(\gamma, r, s) \in\{(1,2,1),(0,4,1)\}$ are provided. Some consequences are also derived.
\end{abstract}

\section{Introduction}

Let $S$ be a closed Riemann surface $S$, Aut $(S)$ be its full group of conformal automorphism and $\operatorname{Aut}^{ \pm}(S)$ be its full group of conformal and anticonformal automorphisms of $S$. If $S$ is either hyperelliptic or has genus $g \in\{0,1,2,3,4\}$, then complete lists for the possibilities for $\operatorname{Aut}(S)$ are known (see, for instance, $[13,14,25]$ ). For $g \geq 5$ such kind of lists are not complete for the non-hyperelliptic case. In the hyperelliptic case, of genus at least two, the order two cyclic group $H$ generated by the hyperelliptic involution is normal subgroup, in particular, $\operatorname{Aut}^{ \pm}(S)$ induces a group of conformal/anticonformal automorphisms of the quotient orbifold $S / H$; the Riemann sphere with exactly $2(g+1)$ special points, all of them of order 2 . As the group of conformal/anticonformal automorphisms of the Riemann sphere is the group of (extended) Möbius transformations, the computation of the induced group can be done explicitly. The above procedure may be done for any closed Riemann surface $S$ admitting a group $H<\operatorname{Aut}(S)$ which is normal in $\operatorname{Aut}^{ \pm}(S)$ (for example, if $H$ is unique in $\operatorname{Aut}(S)$ ). It seems then interesting to provide concrete examples on which we have such a normality (or uniqueness) situation for given algebraic classes of groups $H<\operatorname{Aut}(S)$.

2000 Mathematics Subject Classification: 30F10, 30F35, 30F40.

Keywords: Riemann surfaces, orbifolds, Kleinian groups, automorphisms. 
In this note we consider a class of groups for which the normality property holds. First we need some definitions. An orbifold $\mathcal{O}$ is said to have signature $\left(\gamma, r ; v_{1}, \ldots, v_{r}\right)$ if its underlying topological space is a closed orientable surface of genus $\gamma$, the number of special (or conical) points is exactly $r$ and the branching orders are $v_{1}, \ldots, v_{r}$; if we are not interested on the branching orders $v_{j}$, then we just say that it has type $(\gamma, r)$. A triple $(\gamma, r, s)$ is called admissible if $\gamma, r, s$ are non-negative integers so that $2 \gamma-2+r>0$ and $s \geq 1$. If $p$ is a prime and $s$ some positive integer, then we say that a group $H$ is a $p^{s}$-group if $|H|=p^{s}$. If moreover $S / H$ is an orbifold of signature $(\gamma, r)$, then we say that $H$ is a $p^{s}$-group of type $(\gamma, r)$. In Section 4 we provide a simple and short proof of the following.

Theorem 1. Let $(\gamma, r, s)$ be an admissible triple. Then, there exists a positive integer $B(\gamma, r, s)$, depending only on $\gamma, r$ and $s$, so that, if $p \geq B(\gamma, r, s)$ is a prime, $S$ is a closed Riemann surface of genus at least 2 and $H<\operatorname{Aut}(S)$ is a $p^{s}$-group of type $(\gamma, r)$, then $H$ is the unique such subgroup of $\operatorname{Aut}(S)$ and, in particular, $H$ is a normal subgroup of $\operatorname{Aut}^{ \pm}(S)$.

Theorem 1 may not be a surprise for many of the specialists, but we have not found it explicitly in the literature. Such a result has various applications which we detail in Section 2. Let us fix an admissible triple $(\gamma, r, s)$. Theorem 1 permits us to do the following definitions. Let $q(\gamma, r, s)$ to be the minimal positive integer value ensuring the property that: if $p \geq q(\gamma, r, s)$ is a prime and $S$ is a closed Riemann surface of genus at least 2 and $H<\operatorname{Aut}(S)$ is a $p^{s}$-group of type $(\gamma, r)$, then $H \triangleleft \operatorname{Aut}(S)$; and let $\widehat{q}(\gamma, r, s)$ to be the minimal positive integer value ensuring the property that: if $p \geq \widehat{q}(\gamma, r, s)$ is a prime and $S$ is a closed Riemann surface of genus at least 2 and $H<\operatorname{Aut}(S)$ is a $p^{s}$-group of type $(\gamma, r)$, then $H$ is unique in $\operatorname{Aut}(S)$. By the definition $\widehat{q}(\gamma, r, s) \geq q(\gamma, r, s)$. It seems that in the literature there is not explicit formula for these values. Next, we present only two cases.

Theorem 2. $q(1,2,1)=\widehat{q}(1,2,1)=11, q(0,4,1)=\widehat{q}(0,4,1)=7$.

\subsection{Some examples}

Next, we provide some examples we need in order to obtain the above values.

(1) The classical Klein's curve $S_{3}$ (given by the curve $x^{3} y+y^{3} z+z^{3} x=0$ ) has genus $p=3$ and it has total group of automorphisms of order 168; this being isomorphic to the simple group $P S L(2,7)$. This surface has a cyclic group of order 3 which is non-normal with quotient an orbifold of signature $(1,2 ; 3,3)$ (see also $[21,25]$ ). 
(2) In genus $p=5$ we have a Riemann surface $S_{5}$ with a total group of order 160 (the quotient $S_{5} / \operatorname{Aut}\left(S_{5}\right)$ being of signature $(0,3 ; 2,4,5)$ ) [13]. In this surface we have a conformal automorphism of order 5 which quotient $S_{5}$ to an orbifold of signature $(1,2 ; 5,5)$. The cyclic group $H$ of order 5 generated by such a conformal automorphism cannot be normal in $\operatorname{Aut}\left(S_{5}\right)$. In fact, in such a case, the group $\operatorname{Aut}\left(S_{5}\right)$ will induce a group of order 12 , say $G_{12}$, of conformal automorphisms on the orbifold $S_{5} / H$ that preserves the two branch points (both of order 5). This asserts the existence of an automorphism of order 3 in $G_{12}$ that fixes both of them. This is a contradiction to the fact that such an orbifold quotient by $G_{12}$ must be of signature $(0,3 ; 2,4,5)$.

(3) In genus $p=7$ we have Fricke's curve $S_{7}$ [16] with total group of automorphisms of order 504; this being the simple group $P S L(2,8)$. The quotient $S_{7} / P S L(2,8)$ has signature $(0,3 ; 2,3,7)$. The cyclic group of order 7 is then a non-normal subgroup of $\operatorname{Aut}\left(S_{7}\right)$ with quotient an orbifold of signature $(1,2 ; 7,7)$.

(4) The closed Riemann surface $S_{4}$ of genus 4 (given as the complete intersection of the quadric $x_{1} x_{4}+x_{2} x_{3}=0$ and the cubic $x_{1}^{2} x_{3}+x_{2}^{2} x_{1}+$ $x_{3}^{2} x_{4}+x_{4}^{2} x_{2}=0$ in the 3 -dimensional complex projective space), called the Bring's curve, has $\operatorname{Aut}\left(S_{4}\right) \cong \mathfrak{S}_{5}$, the symmetric group in five letters $\mathfrak{S}_{5}$ (see, for instance, $[1,22]$ ). In this way, we have a cyclic group of order 5 which is non-normal in $\operatorname{Aut}\left(S_{4}\right)$ and whose quotient is an orbifold of signature $(0,4 ; 5,5,5,5)$.

\section{Some Applications}

Corollary 1. Let $(\gamma, r, s)$ be an admissible triple, $p \geq \widehat{q}(\gamma, r, s)$ be a prime, $\mathcal{O}$ be an orbifold of signature $(\gamma, r ; p, \ldots, p)$ and $\mathcal{M}(\mathcal{O})$ be its moduli space. If $S$ is a closed Riemann surface of genus $g$ admitting a $p^{s}$-group of type $(\gamma, r)$, say $H<\operatorname{Aut}(S)$, then $\mathcal{M}(\mathcal{O})$ injects holomorphically into the moduli space $\mathcal{M}_{g}$ of closed Riemann surfaces of genus $g$ as the locus $\mathcal{M}_{g}(H)$ of those classes that admit a conformal action topologically equivalent to $H$.

If $\mathcal{O}$ is an orbifold, then we denote by $\operatorname{Aut}_{\text {orb }}(\mathcal{O})$ (respectively, $\operatorname{Aut}_{\text {orb }}^{ \pm}(\mathcal{O})$ ) the group of orbifold conformal (respectively, conformal and anticonformal) automorphisms of $\mathcal{O}$, that is, the group of conformal (respectively, conformal and anticonformal) automorphisms of the underlying Riemann surface that preserves the special points (branch values) with same order. In this way, if use the notation $\operatorname{Aut}(\mathcal{O})$ (respectively, $\operatorname{Aut}^{ \pm}(\mathcal{O})$ ) to denote the group of conformal (respectively, conformal and anticonformal) automorphisms of the underlying Riemann surface, then $\operatorname{Aut}_{\text {orb }}(\mathcal{O})<\operatorname{Aut}(\mathcal{O})$ 
and $\operatorname{Aut}_{\text {orb }}^{ \pm}(\mathcal{O})<\operatorname{Aut}^{ \pm}(\mathcal{O})$. If $S$ is a closed Riemann surface and $H<$ $\operatorname{Aut}(S)$, then we denote by $\operatorname{Aut}_{H}(S)$ (respectively, $\operatorname{Aut}_{H}^{ \pm}(S)$ ) the normalizer of $H$ in $\operatorname{Aut}(S)$ (respectively, in $\operatorname{Aut}^{ \pm}(S)$ ). If $k \in \operatorname{Aut}_{H}(S)$ (respectively, $k \in \operatorname{Aut}_{H}^{ \pm}(S)-\operatorname{Aut}_{H}(S)$ ), then $k$ induces an (orbifold) conformal (respectively, anticonformal) automorphism of the quotient orbifold $S / H$. An (orbifold) conformal (respectively, anticonformal) automorphism of $S / H$ obtained in this way it is said to lift to $S$. We set Aut $_{\text {orb }}^{H}(S / H)$ (respectively, $\left.\operatorname{Aut}_{o r b}^{ \pm H}(S / H)\right)$ the subgroup of $\operatorname{Aut}_{\text {orb }}(S / H)$ (respectively, $\operatorname{Aut}_{\text {orb }}^{ \pm}(S / H)$ ) consisting of those (orbifold) conformal (respectively, conformal and anticonformal) automorphisms that lift to $S$. Clearly, $\operatorname{Aut}_{\text {orb }}^{H}(S / H) \cong \operatorname{Aut}_{H}(S) / H$ and $\operatorname{Aut}_{\text {orb }}^{ \pm H}(S / H) \cong \operatorname{Aut}_{H}^{ \pm}(S) / H$.

Corollary 2. Let $(\gamma, r, s)$ be an admissible triple, $p$ be a prime number and $S$ be a closed Riemann surface admitting a p $p^{s}$-group $H<\operatorname{Aut}(S)$ of type $(\gamma, r)$. If $p \geq q(\gamma, r, s)$, then $\operatorname{Aut}(S)$ induces a group of (orbifold) conformal automorphisms of $S / H$, and $\operatorname{Aut}(S) / H \cong \operatorname{Aut}_{\text {orb }}^{H}(S / H)$. Also, if $p \geq \widehat{q}(\gamma, r, s)$, then we have that $\operatorname{Aut}^{ \pm}(S)$ induces a group of (orbifold) conformal and anticonformal automorphisms of $S / H$ and $\operatorname{Aut}^{ \pm}(S) / H \cong$ $\mathrm{Aut}_{\text {orb }}^{ \pm H}(S / H)$.

As an example, in Section 6 we compute $\operatorname{Aut}(S)$ in the case of admissible triples $(1,2,1)$.

Corollary 3. Let $S$ be a closed Riemann surface of genus a prime number $p \geq 11$ admitting a conformal automorphism $\eta: S \rightarrow S$ of order $p$. Then,

(i) $S$ is non-hyperelliptic;

(ii) generically, $\operatorname{Aut}(S)=D_{p}$ and

(iii) there is a discrete set of parameters in the corresponding Teichmüller space for which $\operatorname{Aut}(S)=\left\langle u, v: u^{3 p}=v^{2}=1\right.$, vuv $\left.=u^{t p-1}\right\rangle$, where $t=1$ for the case $(3, p-1)=1$ and $t=2$ otherwise. Such a discrete set corresponds to the case when the underlying torus $S /\langle\eta\rangle$ is the one admitting a conformal automorphism of order 6 with fixed points.

Another examples which may we considered are those admitting $H=\mathbb{Z}_{p}^{s}$ as a subgroup of conformal automorphisms so that the quotient $S / H$ is an orbifold of signature $(0, s+1 ; p, \ldots, p)$ (see [5]).

A Fuchsian group $\Gamma<P S L(2, \mathbb{R})$ uniformizing an orbifold of signature $\left(\gamma, t ; m_{1}, \ldots, m_{t}\right)$ will be said to be a Fuchsian group of signature $\left(\gamma, t ; m_{1}, \ldots, m_{t}\right)$.

Corollary 4. Let $(\gamma, r, s)$ be an admissible triple. If $p \geq \widehat{q}(\gamma, r, s)$, then any Fuchsian group $\Gamma$ of signature $(\gamma, r ; p, \ldots, p)$ is uniquely determined by any torsion free normal subgroup of index $p^{s}$. 
Proof. If $\Gamma$ is a Fuchsian group of signature $(\gamma, r ; p, \ldots, p)$, then any torsion free normal subgroup $K \triangleleft \Gamma$ of index $p^{s}$ produces a closed Riemann surface $S$ admitting a group $p^{s}$-group $H$ of conformal automorphisms of type $(\gamma, r)$. If we have two two different Fuchsian groups $\Gamma_{1}$ and $\Gamma_{2}$, both of them with the same signature $(\gamma, r ; p \ldots, p)$, with a common normal subgroup $K$, of in$\operatorname{dex} p^{s}$ in each one, then $K$ will produce a closed Riemann surface $S$ with two different subgroups, say $H_{1}$ and $H_{2}$, so that $\left|H_{j}\right|=p^{s}$ and $S / H_{j}$ is orbifold of type $(\gamma, r)$. If $p \geq \widehat{q}(\gamma, r, s)$, then this a contradiction with the statement of Theorem 1 and the definition of $\widehat{q}(\gamma, r, s)$.

The previous result may be stated, making the suitable modifications, for discrete groups of conformal and anticonformal automorphisms of the hyperbolic plane whose index two orientation-preserving half is a Fuchsian group as above.

In [19] B. Maskit observed that every co-compact torsion free Fuchsian group is uniquely determined by its commutator subgroup. This commutator rigidity property has been extended to the class of torsion free nonelementary function groups [9] and to the more general class of torsion free non-elementary Kleinian groups with an invariant component in its extended region of discontinuity (the union of its region of discontinuity with its double-cusped parabolic fixed points) in [10]. In the presence of torsion, the above rigidity property in general fails for the class of non-elementary Kleinian groups as can be seen in [11]. The following asserts that in some particular classes the presence of torsion do not destroy such a commutator rigidity property.

Corollary 5. Let $r \in\{3,4,5, \ldots\}$ and $p$ be a prime integer so that $r(1-$ $1 / p)>2$ and let $a(r)=\max \{r+1, q(0, r, r-1)\}$. If $p \geq a(r)$ is a prime, then a Fuchsian group of signature $(0, r ; p, \ldots, p)$ is uniquely determined by its commutator subgroup.

Proof. Let us fix the value of $r \geq 3$. The commutator subgroup $\Gamma^{\prime}$ of a group $\Gamma$ as above is necessarily torsion free $[17,15]$ and it uniformizes a closed Riemann surface $S$ of genus $g=\left(2+(r-2) p^{r-1}-r p^{r-2}\right) / 2$. The Fuchsian group $\Gamma$ uniformizes an orbifold $\mathcal{O}$ of signature $(0, r ; p, \ldots, p)$. We have that $H=\Gamma / \Gamma^{\prime} \cong \mathbb{Z}_{p}^{r-1}$ is an Abelian group of conformal automorphisms of $S$ so that $S / H=\mathcal{O}$.

Let us assume we have two groups $\Gamma_{1}$ and $\Gamma_{2}$, with the same presentation as desired, so that they have the same commutator subgroup, say $K$. Let us denote by $S$ the closed Riemann surface uniformized by $K$ and by $\mathcal{O}_{j}$ the orbifold uniformized by $\Gamma_{j}$, for $j=1,2$. In this case, we have two Abelian groups $H_{j} \cong \mathbb{Z}_{p}^{r-1}$ acting on $S$ so that $S / H_{j}=\mathcal{O}_{j}$. If we assume $p \geq q(0, r, r-1)$, then Theorem 1 asserts that both $H_{1}$ and $H_{2}$ are normal 
subgroups of $\operatorname{Aut}(S)$. In order to prove our result we only need to verify that $H_{1}=H_{2}$. If $p \geq a(r)$ and $H_{1} \neq H_{2}$, then we have some $h \in H_{2}-H_{1}$ of order $p$. As $H_{1}$ is normal subgroup, we have that $h$ induces an automorphism $h^{*}$ (as orbifold) of order $p$ of $\mathcal{O}_{1}$. But $h^{*}$ should preserve the $r$ special points of order $p$ on $\mathcal{O}_{1}$, a contradiction to the fact that $p>r$.

\section{Generalities}

In this section we recall some general facts and fix some notations we need in this note. As consequence of results of L. Keen [12] a Fuchsian group uniformizing an orbifold of signature $\left(h, t ; m_{1}, \ldots, m_{t}\right)$ has a presentation of the form

$$
\begin{gathered}
\Gamma=\left\langle a_{1}, \ldots, a_{h}, b_{1}, \ldots, b_{h}, x_{1}, \ldots, x_{t}:\right. \\
\left.x_{1}^{m_{1}}=\cdots=x_{t}^{m_{t}}=\prod_{i=1}^{h} a_{i} b_{i} a_{i}^{-1} b_{i}^{-1} \prod_{j=1}^{t} x_{j}=1\right\rangle, \\
\text { where } \quad m_{1}, \ldots, m_{t} \in\{2,3,4, \ldots\} .
\end{gathered}
$$

We use the notation $G_{\left(h ; m_{1}, \ldots, m_{t}\right)}$ to denote a group with presentation as in (3.1). If $h=0$, we just use the notation $G_{\left(m_{1}, \ldots, m_{t}\right)}$. We denote by $\Delta_{(a, b, c)}$ a Fuchsian group of signature $(0,3 ; a, b, c)$.

In [24], D. Singerman consider the (reduced) Teichmüller space $T^{\sharp}(G)$, where $G$ is a finitely generated Fuchsian. The space $T^{\sharp}(G)$ has the structure of a real analytic variety of dimension $d(G)<\infty$. If $G$ has signature $\left(h ; m_{1}, \ldots m_{s}\right)$, then $d(G)=6 h-6+2 s$. Another of the known main facts, also referenced in [24], asserts that if we consider two finitely generated Fuchsian groups, say $G_{1}$ and $G_{2}$, so that $G_{1}<G_{2}$ with finite index, then $T^{\sharp}\left(G_{2}\right)$ can be embedded as a closed real analytic sub-variety of $T^{\sharp}\left(G_{1}\right)$; in particular, $d\left(G_{2}\right) \leq d\left(G_{1}\right)$. We say that $G_{1}$ has Fuchsian growth to the group $G_{2}$, if $G_{1}<G_{2}$ with finite index. In [24] D. Singerman studies and classifies the Fuchsian growths $G_{1}<G_{2}$ so that $d\left(G_{2}\right)=d\left(G_{1}\right)$.

The moduli space $\mathcal{M}_{g}$ is defined as the space of holomorphic equivalence classes of Riemann surfaces of genus $g$. This space is an analytic space whose (orbifold) universal cover is the Teichmüller space of genus $g$, which, for $g \geq 2$, is isomorphic to the Teichmüller space of a Fuchsian group that uniformizes a closed Riemann surface of genus $g$ (see, for instance, [20] for generalities on Teichmüller and moduli spaces).

We also need the moduli space of orbifolds. If we have orbifolds $\mathcal{O}_{1}$ and $\mathcal{O}_{2}$, then we say that they are holomorphically equivalent if there is a conformal homeomorphism between the underlying Riemann surface structures preserving the special points and their orders. As in the cases in here 
considered all special points have the same order, the conformal homeomorphism is only required to preserve the special points. If we fix the type of an orbifold $\mathcal{O}$, then we have associated the moduli space $\mathcal{M}(\mathcal{O})$ of orbifolds of such types whose points are the holomorphic equivalent classes of orbifolds of the fixed type.

Let $S$ be a closed Riemann surface of genus $g \geq 2, H$ be a cyclic group of conformal automorphisms of $S$ and set $\mathcal{O}=S / H$. Denote by $\mathcal{M}_{g}(H)$ the locus in $\mathcal{M}_{g}$ (the moduli space of genus $g$ ) consisting of classes of Riemann surfaces admitting a group of conformal automorphisms which is topologically equivalent to $H$. We have that $\mathcal{M}_{g}(H)$ is an irreducible subvariety of $\mathcal{M}_{g}$ of dimension $3 \gamma-3+r$, where $\mathcal{O}$ has type $(\gamma, r)$, [3, 4]. In the general situation, that is when $H$ is any (finite) group of conformal automorphisms of a closed Riemann surface $S$ of genus $g$, then the above asserts that $\mathcal{M}_{g}(H)$, the locus in $\mathcal{M}_{g}$ consisting of classes of Riemann surfaces admitting a group of conformal automorphisms which is topologically equivalent to $H$, is an irreducible algebraic variety of complex dimension $3 \gamma-3+r$, where $S / H$ has type $(\gamma, r)$. The moduli space $\mathcal{M}_{g}(H)$ is the normalization of $\mathcal{M}(\mathcal{O})$ and in general they are not equivalent.

Let $(\gamma, r, s)$ be an admissible triple and $S$ be a closed Riemann surfaces admitting a $p^{s}$-group of conformal automorphisms of type $(\gamma, r)$. RiemannHurwitz's formula [2] asserts that $S$ must have genus

$$
g(p)=p^{s}(\gamma-1)+1+\frac{1}{2} \sum_{j=1}^{r} p^{s-l_{j}}\left(p^{l_{j}}-1\right)
$$

for certain values $l_{j} \in\{1, \ldots, s\}$. Let us denote by $X_{\gamma, r, s, p} \subset \mathcal{M}_{g(p)}$ the locus in moduli space consisting of the classes of closed Riemann surfaces of genus $g(p)$ admitting a $p^{s}$-group $H$ of conformal automorphisms of type $(\gamma, r)$. We have that $X_{\gamma, r, s, p}$ is an algebraic variety, of dimension $3 \gamma-3+r$, which is a finite union of irreducible subvarieties of $\mathcal{M}_{g(p)}$; the different components corresponding to the different topological actions of $H$ (if the topological action of the group $H$ is rigid, then such a locus has only one component). These components may or not intersect (or self-intersect) inside the moduli space. The main result of this paper asserts in particular that if $p$ is sufficiently large in comparison to $\gamma, r$ and $s$, then they do not (self-) intersect.

If we have a conformal automorphism $h$ of some Riemann surface $S$ and $x \in S$ is a fixed point of $h$, then there is a unique value $\alpha \in[-\pi, \pi)$ so that in local coordinates $h(z)=e^{i \alpha} z$; such a value is called the rotation number of $h$ about $x$. 


\section{Proof of Theorem 1}

Let $S$ be a closed Riemann surface (uniformized by the hyperbolic plane) admitting a $p^{s}$-group $H$ as group of conformal automorphisms, where $p \geq 3$ is a prime, of type $(\gamma, r)$, We denote by $\mathcal{O}$ the quotient orbifold $S / H$. KoebePoincaré's uniformization theorem asserts the existence of a co-compact torsion free Fuchsian group $\Gamma$ so that $S=\mathbb{H}^{2} / \Gamma$. If we denote by $N[\Gamma]$ the normalizer of $\Gamma$ in the group of conformal automorphisms of $\mathbb{H}^{2}$, then we have that $N[\Gamma]$ is again a co-compact Fuchsian group [18] maybe with torsion so that $\operatorname{Aut}(S)=N[\Gamma] / \Gamma$ and $S / \operatorname{Aut}(S)=\mathbb{H}^{2} / N[\Gamma]$. Also there is a group $\Gamma_{1}$ so that $\Gamma \triangleleft \Gamma_{1}<N[\Gamma], H=\Gamma_{1} / \Gamma$ and $\mathcal{O}=\mathbb{H}^{2} / \Gamma_{1}$. We set $n_{1}(0 ; r)=r+1$, $n_{1}(1 ; r)=\max \{5, r+1\}$ and, for $\gamma \geq 2, n_{1}(\gamma, r)=2 \gamma+2$, for $\gamma \geq 2$. The choice we have made of $n_{1}(\gamma, r)$ ensures that if $p \geq n_{1}(\gamma, r)$ is a prime, then no orbifold of type $(\gamma, r)$ admits an orbifold automorphism of order $p$. In fact, note that very (orbifold) conformal automorphism of an orbifold of type $(\gamma, r)$ is a conformal automorphism of the underlying closed Riemann surface of genus $\gamma$ that permutes the $r$ special conical points. In this way, the choice of $n_{1}(0 ; r)$ is clear.

As conformal automorphism of prime order $p \geq 5$ acts free fixed points on a genus one Riemann surface, the choice for $n_{1}(1 ; r)$ is correct. In the case that $\gamma \geq 2$, as a consequence of Riemann-Hurwitz formula, if $h$ is a conformal automorphism of order $p$ prime on a closed Riemann surface of genus $\gamma \geq 2$, then $p \leq 2 \gamma+1$.

Lemma 1. If $p \geq n_{1}(\gamma, r)$ is a prime so that $p^{s}$ divides $|\operatorname{Aut}(S)|$, then $|\operatorname{Aut}(S)|=a p^{s}$, where $a \in \mathbb{N}$ is relative prime to $p$.

Proof. Assume $|\operatorname{Aut}(S)|=a p^{s+1}$, where $a \in \mathbb{N}$. Sylow's theorem asserts the existence of a group $K_{p}<\operatorname{Aut}(S)$ so that $\left|K_{p}\right|=p^{s+1}$ and $H \triangleleft K_{p}$. In particular, this asserts that on the orbifold $S / H$ should be an (orbifold) automorphism of order $p \geq n_{1}(\gamma, r)$, a contradiction.

As a consequence of lemma 1 , for $p \geq n_{1}(\gamma, r)$ the $p$-Sylow subgroups of $\operatorname{Aut}(S)$ are groups of order $p^{s}$, all of them conjugate to $H$. The number of possible different $p$-subgroups of $\operatorname{Aut}(S)$ has the form $N_{p}=(1+k p)$, certain $k \geq 0$ and, in particular, the order of $\operatorname{Aut}(S)$ may be written as $|\operatorname{Aut}(S)|=$ $b p^{s} N_{p}$, where $(p, b)=1$. We assume from now on that $p \geq n_{1}(\gamma, r)$. If we are able to find a value $n_{2}(\gamma, r) \geq n_{1}(\gamma, r)$ so that for $p \geq n_{2}(\gamma, r)$ we have $k=0$, then we will be done with the proof.

The group $N[\Gamma]$ has a canonical presentation as in (3.1). By RiemannHurwitz's formula

$$
|\operatorname{Aut}(S)|=\frac{2(g(p)-1)}{M[N[\Gamma]]},
$$


where

$$
M[N[\Gamma]]=2(h-1)+\sum_{j=1}^{t}\left(1-\frac{1}{m_{j}}\right)
$$

and $g(p)$ as in equation (3.2). If we set $A(\gamma, r)=\gamma+r / 2-1$, then $g(p)-1 \leq$ $A(\gamma, r) p^{s}$ and, in particular,

$$
b p^{s} N_{p}=|\operatorname{Aut}(S)| \leq \frac{2 A(\gamma, r) p^{s}}{M[N[\Gamma]]}, \quad(p, b)=1 .
$$

On the other hand, the minimum value that $M[N[\Gamma]]$ may have is $1 / 42$. It follows from (4.1) that $b N_{p} \leq 84 A(\gamma, r)$. If $k>0$, that is $N_{p} \geq 1+p$, then, as $b \geq 1$, the last inequality obligates to have $p \leq 84 A(\gamma, r)-1$. In this way, if we choose $n_{2}(\gamma, r)=\max \left\{n_{1}(\gamma, r), 84 A(\gamma, r)\right\}$, then the above asserts that for primes $p \geq n_{2}(\gamma, r)$ we have $N_{p}=1$ as desired and, in particular, $|\operatorname{Aut}(S)|=b p^{s}$, where $(b, p)=1$. This finishes the proof of Theorem 1 .

\section{Proof of Theorem 2}

Let $S$ be a closed Riemann surface admitting a group $H \cong \mathbb{Z}_{p}$ as group of conformal automorphisms, where $p \geq 3$ is a prime, so that $S / H$ is an orbifold $\mathcal{O}$ of type $(\gamma, r)$, where $(\gamma, r) \in\{(0,4),(1,2)\}$. Let $\Gamma$ and $\Gamma_{1}$ be Fuchsian groups acting on the hyperbolic plane $\mathbb{H}^{2}$ so that $\mathbb{H}^{2} / \Gamma=S, \mathbb{H}^{2} / \Gamma_{1}=S / H$ and $\Gamma \triangleleft \Gamma_{1}$ of index $p$. If $N[\Gamma]$ is the normalizer of $\Gamma$ in the group of conformal automorphisms of $\mathbb{H}^{2}$, then $N[\Gamma]$ is a co-compact Fuchsian group, maybe with torsion, so that $\operatorname{Aut}(S)=N[\Gamma] / \Gamma, S / \operatorname{Aut}(S)=\mathbb{H}^{2} / N[\Gamma]$ and $\Gamma \triangleleft \Gamma_{1}<N[\Gamma]$. We need to prove that for prime integers $p \geq q(\gamma, r, 1)$ it holds that $\Gamma_{1} \triangleleft N[\Gamma]$ and that for primes values of $p<q(\gamma, r, 1)$ the normality property fails. In our cases $n(\gamma, r)=5$. It follows from Lemma 1 that $b p N_{p}=|\operatorname{Aut}(S)|=2(p-h) / M[N[\Gamma]]$, for some $b \in\{1,2,3, \ldots\},(b, p)=1$, where $N_{p}=(1+k p)$ stands for the number of possible different $p$-Sylow subgroups of $\operatorname{Aut}(S), h=1$ for $(\gamma, r)=(1,2)$ and $h=2$ for $(\gamma, r)=(0,4)$.

5.1. $q(1,2,1) \geq 11$.

Let $p \geq 11$ be a prime. As $\Gamma_{1}$ has signature $(1,2 ; p, p)$, then $d\left(\Gamma_{1}\right)=4$, in particular, $d(N[\Gamma]) \in\{0,2,4\}$. If $d(N[\Gamma])=4$, then $N[\Gamma]$ has signature $(0,5 ; 2,2,2,2, p)[24]$. In this case $\left[N[\Gamma]: \Gamma_{1}\right]=2$, so $\Gamma_{1} \triangleleft N[\Gamma]$. If $d(N[\Gamma])=2$, then $6 g-6+2 r=2$. We need to consider two cases.

(i) If $g=r=1$, then [24] the only possible Fuchsian growth of $\Gamma_{1}$ contains it as an index two, then as a normal subgroup. 
(ii) If $g=0$ and $r=4$, then

$$
b(1+k p) p=\frac{2(p-1)}{2-\frac{1}{m_{1}}-\frac{1}{m_{2}}-\frac{1}{m_{3}}-\frac{1}{\rho p}} \leq \frac{2(p-1)}{\frac{1}{2}-\frac{1}{p}} .
$$

The term $\frac{1}{\rho p}$ in the formula (5.1) is due to the fact that in a Fuchsian group the finite order elements are conjugate to powers of the finite order elements in a canonical presentation. As $b \geq 1, p \geq 11$ and $k \geq 0$ is integer, the above asserts that necessarily $k=0$. It follows that there is a unique Sylow $p$-subgroup, so $H \triangleleft \operatorname{Aut}(S)$.

If $d(N[\Gamma])=0$, then $N[\Gamma]$ is a triangular group and

$$
b(1+k p) p=\frac{2(p-1)}{1-\frac{1}{m_{1}}-\frac{1}{m_{2}}-\frac{1}{\rho p}} \leq \frac{2(p-1)}{\frac{1}{6}-\frac{1}{p}} .
$$

If $p \geq 17$, then $k=0$ and, in particular, $H \triangleleft \operatorname{Aut}(S)$. Let us assume now that $p \in\{11,13\}$. We search for all possible combinations of $m_{1}, m_{2}$ and $\rho$ for some $k \geq 1$ satisfying the equality (5.2). We obtain that the only possibility happens for $p=11$; in which case $N[\Gamma]$ has signature $(0,3 ; 2,3,11)$ and $|\operatorname{Aut}(S)|=264$. We now proceed to show that this case is not possible. In fact, let us assume the existence of this possibility. It is known that every group of order 264 is not simple; moreover, no divisor of 264 can be the order of some non-abelian simple group. This asserts that we may construct a non-trivial chain $K_{1} \triangleleft K_{2} \triangleleft \cdots K_{N} \triangleleft \operatorname{Aut}(S)$, so that $\operatorname{Aut}(S) / K_{N}$ is a nontrivial abelian group. But, as the group $N[\Gamma]$ has signature $(0,3 ; 2,3,11)$, then we have $N[\Gamma]=N[\Gamma]^{\prime}$, where $N[\Gamma]^{\prime}$ denotes the commutator subgroup of $N[\Gamma]$, a contradiction to the existence of the above chain. All the above asserts that $q(1,2,1) \leq 11$. Examples (1), (2) and (3) of Section 1.1 permit us to obtain $q(1,2,1)=11$

\section{2. $q(0,4,1) \geq 7$}

Let us assume $p \geq 7$. The arguments are similar to the previous case. As $d\left(\Gamma_{1}\right)=1$, then $d(N[\Gamma]) \in\{0,1\}$. If $d(N[\Gamma])=1$, then by [24], there are only normal Fuchsian growths of $\Gamma_{1}$. Let us assume now on $d(N[\Gamma])=0$, then

$$
b(1+k p) p=(2 p-4) /\left(1-\frac{1}{k_{1}}-\frac{1}{k_{2}}-\frac{1}{\rho p}\right), \quad \rho \in\{1,2, \ldots\} .
$$

As $p \geq 7,(5.3)$ asserts $k \leq f(p)=\frac{12(p-2)}{p(5 p-6)}$ and, in particular, for $p \geq 17$, we get $k=0$. We need to consider the cases $p \in\{7,11,13\}$. 
5.2.1. Case: $p \in\{11,13\}$

We only need to consider the case $k=1$. As $b \leq 12(p-2) /((p+1)(5 p-6))$, then $b=1$. By direct inspection, since $\rho$ is a positive integer, we only need to take care of the case $p=11, k_{1}=2$ and $k_{2}=3$; in which case $\rho=3$ and $N[\Gamma] \cong \Delta_{(2,3,33)}$. The triangular group $\Delta_{(2,3,33)}$ contains no no-normal subgroup of signature $(0,4 ; 11,11,11,11)[15]$ and the only normal subgroups of $\Delta_{(2,3,33)}$ have signature $(0,4 ; 2,2,2,11)$, all of them of index 3 . If we consider one of them, say $G_{(2,2,2,11)}$, then $\Gamma_{1} /\left(\Gamma_{1} \cap G_{(2,2,2,11)}\right)<\Delta_{(2,3,33)} / G_{(2,2,2,11)} \cong \mathbb{Z}_{3}$. (i) If $\Gamma_{1}=\Gamma_{1} \cap G_{(2,2,2,11)}$, then $\Gamma_{1}<G_{(2,2,2,11)}$. As $\Gamma_{1}$ is the commutator subgroup of $G_{(2,2,2,11)}[15]$ and $G_{(2,2,2,11)}$ is normal subgroup of $\Delta_{(2,3,33)}, \Gamma_{1}$ is normal subgroup of $\Delta_{(2,3,33)}$. This is a contradiction to the fact that signature of $\Gamma_{1}$ is not of the form $(0,4 ; 2,2,2,11)$. (ii) If $\left[\Gamma_{1}: \Gamma_{1} \cap G_{(2,2,2,11)}\right]=3$, then the commutator subgroup of $\Gamma_{1}$ is a normal subgroup of $\Gamma_{1} \cap G_{(2,2,2,11)}$, a contradiction to the fact that the commutator subgroup of $\Gamma_{1}$ has index $11^{3}$ in $\Gamma_{1}$.

\subsubsection{Case: $p=7$ and $k \geq 1$}

In this case, $b(1+7 k) \leq 60$. If we assume equality, then $(b, k)=(4,2)$ and it follows that $N[\Gamma]=\Delta_{(2,3,7)}$, in particular, $|\operatorname{Aut}(S)|=420$ and $\operatorname{Aut}(S)$ must contain 15 7-Sylow subgroups. As there is no simple group of order 420, there is a non-trivial normal subgroup $H_{0} \triangleleft \operatorname{Aut}(S)$, in particular, $H_{0}$ cannot be of order 7 ; so $\left|\operatorname{Aut}(S) / H_{0}\right| \neq 60$. Now, if we check at all possible orders of $\operatorname{Aut}(S) / H_{0}$, we obtain that $\operatorname{Aut}(S) / H_{0}$ is either non-simple or Abelian. In this way we may construct a non trivial chain of normal subgroups $H_{0} \triangleleft$ $H_{1} \ldots \triangleleft H_{N} \triangleleft \operatorname{Aut}(S)$, so that $\operatorname{Aut}(S) / H_{N}$ is a non-trivial Abelian group. As $\Delta_{(2,3,7)}$ is its own commutator subgroup, we get a contradiction. As a consequence, $b(1+7 k)<60$, then $b(1+7 k) \leq 15$ and, in particular, $k \in\{1,2\}$ and $b=1$.

If $k=1$, as $\rho$ is a positive integer, then the only case we need to take care is $k_{1}=2$ and $k_{2}=4$. In this situation $\rho=2$ and $N[\Gamma] \cong \Delta_{(2,4,14)}$. The only normal subgroups of $\Delta_{(2,4,14)}$ have signature $(0,4 ; 2,2,2,7)$, all of them of index two. Let one of them be $G_{(2,2,2,7)}$. In this way $\Gamma_{1} /\left(\Gamma_{1} \cap G_{(2,2,2,7)}\right)<$ $\Delta_{(2,4,14)} / G_{(2,2,2,7)} \cong \mathbb{Z}_{2}$. (i) If $\Gamma_{1}=\Gamma_{1} \cap G_{(2,2,2,7)}$, then $\Gamma_{1}<G_{(2,2,2,7)}$. As $\Gamma_{1}$ is the commutator of $G_{(2,2,2,7)}[15]$, and, as $G_{(2,2,2,7)}$ is normal in $\Delta_{(2,4,14)}$, we have that $\Gamma_{1}$ is normal subgroup of $\Delta_{(2,4,14)}$. This is a contradiction to the fact that signature of $\Gamma_{1}$ is not of the form $(0,4 ; 2,2,2,7)$. (ii) If $\left[\Gamma_{1}: \Gamma_{1} \cap G_{(2,2,2,7)}\right]=2$, then the commutator subgroup of $\Gamma_{1}$ is a normal subgroup of $\Gamma_{1} \cap G_{(2,2,2,7)}$, a contradiction to the fact that the commutator subgroup of $\Gamma_{1}$ has index $7^{3}$ in $\Gamma_{1}$. 
If $k=2$, then we only need to take of the case $k_{1}=2$ and $k_{2}=3$, so $\rho=2$ and $N[\Gamma] \cong \Delta_{(2,3,14)}$. This obligates to $\Delta_{(2,3,14)}$ to contain the group $\Gamma_{1}$ with index 15 , in particular, there is a homomorphism $\theta: \Delta_{(2,3,14)} \longrightarrow \mathfrak{S}_{15}$, where $\mathfrak{S}_{15}$ is the permutation group in 15 letters, so that $\theta\left(\Delta_{(2,3,14)}\right)$ is transitive. Choose $x_{1} \in \Delta_{(2,3,14)}$ so that $x_{1}^{2}=1$ and $x_{1}$ is primitive. We have that $\theta\left(x_{1}\right)$ should fix a letter, in particular, it follows that $\Gamma_{1}$ must contain an element of order 2, a contradiction. All the above asserts that $q(0,4,1) \leq 7$. Example (4) of Section 1.1 asserts $q(0,4,1)=7$.

\section{3. $q(1,2,1)=\widehat{q}(1,2,1)$ and $q(0,4,1)=\widehat{q}(0,4,1)$}

As $\widehat{q}(\gamma, r, s) \geq q(\gamma, r, s)$, we have that $\widehat{q}(1,2,1) \geq 11$. If we have two different groups $H_{1}, H_{2}<\operatorname{Aut}(S), H_{j} \cong \mathbb{Z}_{p}, S / H_{j}$ of type $(1,2)$, for some prime integer $p \geq 11$, then both of them are normal subgroups of $\operatorname{Aut}(S)$. In this way, $\mathrm{H}_{2}$ induces a cyclic group of order $p$ as a group of orbifold conformal automorphisms of $S / H_{1}$, a contradiction; obtaining that $\widehat{q}(1,2,1)=11$. Similar arguments show that $\widehat{q}(0,4,1)=7$.

\section{Proof of Corollary 3}

Let us consider a closed Riemann surface $S$ of genus $p \in\{2,3,4, \ldots\}$ admitting a conformal automorphism $\eta: S \rightarrow S$ of order $p$ (at this moment not necessarily prime) so that (i) $\operatorname{Fix}(\eta)=\operatorname{Fix}\left(\eta^{l}\right)=\{a, b\}$, for $l=1,2, \ldots, p-1$; and (ii) the rotation number of $\eta$ about $a$ is opposite to the rotation number of $\eta$ about $b$ (this is the situation when $p$ is a prime number). Let $\pi: S \rightarrow S / H$ be the branched covering induced by the action of the cyclic group $H=\langle\eta\rangle \cong \mathbb{Z}_{p}$, and $K<\operatorname{Aut}_{\text {orb }}(S / H)$ be the subgroup whose elements are the automorphisms that lift to $S$ under $\pi$. On $S / H$ there is a (not unique) simple geodesic arc $\gamma$ (which we fix now on) having as its end points the two branch values $\pi(a)$ and $\pi(b)$, so that $\pi^{-1}(S / H-\gamma)$ consists on exactly $p$ components, each one homeomorphic to $S / H-\gamma$ under $\pi$. We have that $\pi^{-1}(\gamma)$ is homeomorphic to a connected graph consisting on two vertices ( $a$ and $b$ ) and $p$ edges connecting them (see figure 1). If $t \in \operatorname{Aut}_{\text {orb }}(S / H)$ preserves the arc $\gamma$, then it lifts, that is, $t \in K$. Unfortunately, the arc $\gamma$ is not unique and it may happen that an automorphism belongs to $K$ and does not preserve $\gamma$. It is not hard to see that we may find a conformal involution (with 4 fixed points) $\tau_{1}: S / H \rightarrow S / H$ permuting both branch values so that $\tau_{1}(\gamma)=\gamma^{-1}$; that is, $\tau_{1} \in K$. If we lift this involution to $S$ we obtain a conformal automorphism $\tau$ so that $\tau^{2} \in H=\langle\eta\rangle$ that should permute both fixed points of the conformal automorphism $\eta$ of order $p$. As the rotation number of $\eta$ at these two fixed points are opposite $\tau$ cannot 
commute with $\eta$. Also, as $\tau$ must conjugate $\eta$ in either $\eta$ or $\eta^{-1}$ (as it should preserve the absolute value of angle of rotation) we have that $(\tau \eta)^{2}=1$. As $\tau_{1}$ has fixed points which are different from the branch values, $\tau$ may be chosen to have fixed points which are different from those of $\eta$. If $\tau^{2} \neq 1$, then a non-trivial power of $\eta$ with different fixed points as $\eta$ exists, a contradiction to our assumptions. It follows that $\tau^{2}=1$ and, in particular, the group $D_{p}=\langle\eta, \tau\rangle$ is the dihedral group of order $2 p$ (already observed by Ries in [23]).

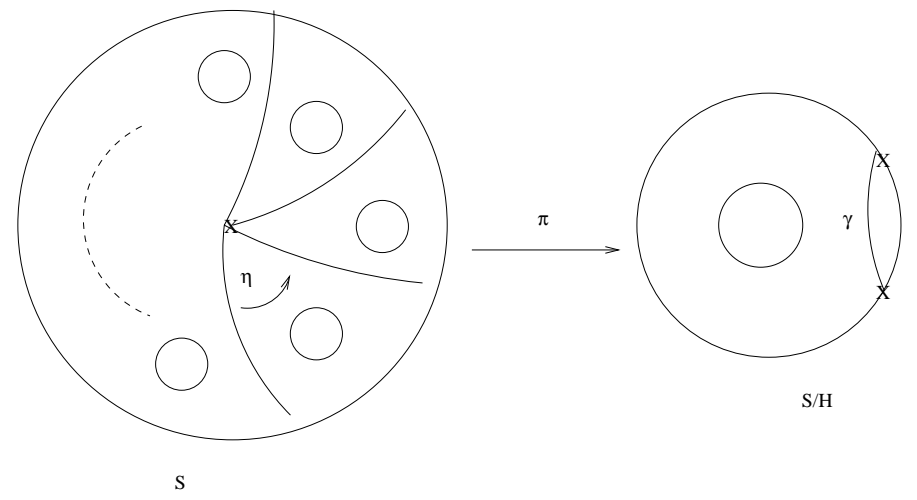

Figure 1

As generically an orbifold of signature $(1,2 ; p, p)$ has no more automorphisms other than the involution $\tau_{1}$, the subgroup $K$ is generically $\left\langle\tau_{1}\right\rangle$. In particular, if $p \geq 11$ is a prime number, then Theorem 2 asserts that generically $\operatorname{Aut}(S)=D_{p}$ and that there are exactly two possible ways to obtain an extra automorphism in $K$.

(a) Both branch values are permuted by a conformal involution without fixed points or

(b) The induced genus one surface has automorphisms with fixed points of order 3, 4 or 6 and preserve the branch values.

Any simple loop $\alpha \subset S / H$, disjoint from $\gamma$ lifts to exactly $p$ pairwise disjoint simple loops. Also, if a simple loop $\alpha \subset S / H$, disjoint from the two branch values, intersects transversally $\gamma$ at exactly one point, then $\pi^{-1}(\alpha)$ has exactly one component (then invariant under $\eta$ ). In this way, if $h \in$ Aut $_{\text {orb }}(S / H)$ and $\alpha \in S / H$ is a simple loop disjoint from $\gamma$ so that $h(\alpha)$ intersects $\gamma$ (transversally) exactly at one point, then we have that $h \notin K$. This observation permits us to see that if both branched values are permuted by a conformal involution $h$, acting free fixed points on $S / H$, then $h \notin K$. The reason of this is that we may find a simple loop $\alpha \subset S / H$, disjoint form $\gamma$ so that $h(\alpha)$ intersects $\gamma$ (transversally) exactly at one point. In this way, case (a) does not give extra automorphisms in $K$. In a similar way, if $h$ is a 
conformal involution that fixes each of the two branch values, then $h \notin K$, taking care of one case in (b). In particular, we have obtained part (i) and (ii) of Corollary 3. Now, to obtain part (iii) of the Corollary we assume the torus $S / H$ admits a conformal automorphism $\rho$ of order 6 with fixed points (it has exactly one fixed point, its square has exactly three fixed points and its cube has exactly four fixed points). In this case, as the two branch values of $S / H$ are invariant under $\rho$ and $\rho$ has exactly one fixed point, we must have that it permutes them, that is, the automorphism $h=\rho^{2}$, of order 3 , has them as two of its three fixed points. In this case, $S / \operatorname{Aut}(S)=(S / H) /\langle\rho\rangle$ is the orbifold of signature $(0 ; 2,6,3 p)$. Let us consider the Fuchsian group $G$ that uniformizes our orbifold $S / H$, which has the presentation as follows

$$
G=\left\langle a, b, x_{1}, x_{2}: x_{1}^{p}=x_{2}^{p}=a b a^{-1} b^{-1} x_{2}^{-1} x_{1}^{-1}=1\right\rangle .
$$

Let us consider the external automorphism $\theta: G \rightarrow G$ defined as (see figure 2) $\theta(a)=b, \theta(b)=b^{-1} a^{-1} x_{1}, \theta\left(x_{1}\right)=a^{-1} x_{1} a$ and $\theta\left(x_{2}\right)=a^{-1} b^{-1} x_{2} b a$, which is geometrically induced by the automorphism $h$.

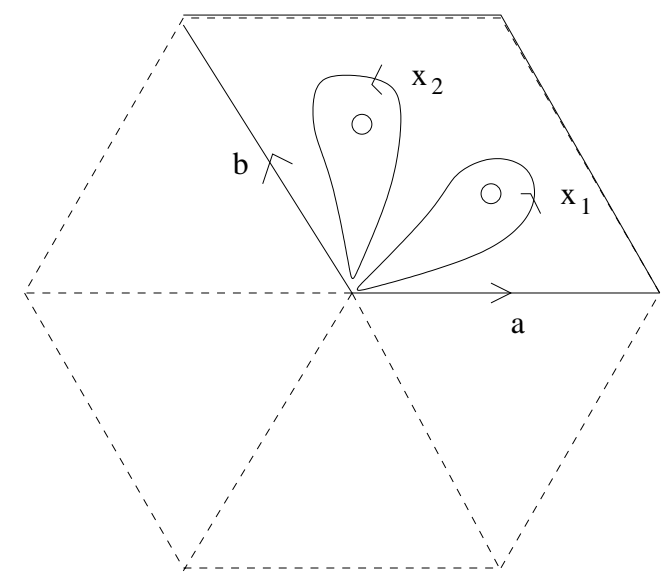

FIGURE 2

The branched cyclic covering $\pi: S \rightarrow S / H$ is defined by the smaller normal subgroup $\mathcal{N}$ of $G$ containing $x_{1}^{k} a$ and $x_{1}^{k} b$, where $3 k \equiv-1 \bmod p$. Clearly, $\theta$ leaves invariant $\mathcal{N}$, in particular, $h \in K$. Now, we have an automorphism $\zeta: S \rightarrow S$, a lifting of the automorphism $h: S / H \rightarrow S / H$, so that $\langle\zeta, \eta\rangle=$ $\langle\psi\rangle \cong \mathbb{Z}_{3 p}$ and $\psi: S \rightarrow S$ has the same fixed points as for $\eta$. We proceed to give a concrete description of the group $\operatorname{Aut}(S)$ in this case. Let us consider an orbifold $\mathcal{O}_{0}$ of signature $(0 ; 2,6,3 p)$, Köbe's uniformization theorem gives us a Fuchsian uniformization of $\mathcal{O}_{0}$ by a Fuchsian group

$$
K_{0}=\left\langle U, V: U^{3 p}=V^{2}=(V U)^{6}=1\right\rangle .
$$


A fundamental domain $P_{0}$ of $K_{0}$ is given by a hyperbolic triangle with angles $2 \pi / 3 p, \pi / 6$ and $\pi / 6$. The transformation $V$ is given by the involution with fixed point at the middle point of the side of $P_{0}$ determined by the two vertices with angle $\pi / 6$, and $U$ is given by the elliptic element fixing the vertex with angle $2 \pi / 3 p$ and sending one of the sides at such a vertex to the other. Let us set $S=U^{3}, W=U V U^{-1}, R=U^{2} V U^{-2}, \widehat{S}=W S W$, $A=W R, B=W V$, and consider the subgroups

$$
\left\{\begin{aligned}
& K_{1}=\left\langle S, V, W, R: S^{p}=V^{2}=W^{2}=R^{2}=(V W R S)^{2}=1\right\rangle \\
& K_{2}=\left\langle S, \widehat{S}, A, B: S^{p}=\widehat{S}^{p}=S B^{-1} A^{-1} \widehat{S} B A=1\right\rangle \\
& K_{3}=\left\langle S \widehat{S}, S(S \widehat{S}) S^{-1}, \ldots, S^{-1}(S \widehat{S}) S, S^{k} A, S\left(S^{k} A\right) S^{-1}, \ldots\right. \\
&\left.S^{-1}\left(S^{k} A\right) S, S^{l} B, S\left(S^{l} B\right) S^{-1}, \ldots, S^{-1}\left(S^{l} B\right) S\right\rangle .
\end{aligned}\right.
$$

It is clear that $K_{2} \triangleleft K_{1}$ of index two. The group $K_{1}$ is normal subgroup of index three in $K_{0}$ and uniformizes an orbifold $\mathcal{O}_{1}$ of signature $(0 ; 2,2,2,2, p)$. In fact, a fundamental domain $P_{1}$ for $K_{1}$ can be obtained by $P_{1}=P_{0} \cup$ $U\left(P_{0}\right) \cup U^{2}\left(P_{0}\right)$. To get normality, we only need to observe that $U S U^{-1}=S$, $U V U^{-1}=V, U W U^{-1}=R$ and $U R U^{-1}=S V S^{-1}$.

The group $K_{2}=\left[K_{0}, K_{0}\right]$ is a normal subgroup of index six in $K_{0}$ and uniformizes an orbifold of signature $(1,2 ; p, p)$. In fact, a fundamental domain $P_{2}$ for $K_{2}$ is given by $P_{2}=P_{1} \cup W\left(P_{1}\right)$, in particular, we have that $K_{2}$ has index two in $K_{1}$, then of index 6 in $K_{0}$. We also have that $U S U^{-1}=S$, $U \widehat{S} U^{-1}=A^{-1} \widehat{S} A, U A U^{-1}=A^{-1} \widehat{S} B S^{-1}, U B U^{-1}=A^{-1}, V S V=B^{-1} \widehat{S} B$, $V \widehat{S} V=B^{-1} S B, V A V=B^{-1} A^{-1} B$ and $V B V=B^{-1}$, providing the normality. As $K_{0} / K_{2} \cong \mathbb{Z}_{6}$, we have that $K_{0}^{\prime}$, the commutator subgroup of $K_{0}$, is a subgroup of $K_{2}$. Since, $K_{0} / K_{0}^{\prime} \cong \mathbb{Z}_{6}$, we obtain the equality $K_{2}=K_{0}^{\prime}$.

If $l+k \equiv 0 \bmod p$ and $3 l+2 \equiv 0 \bmod p$, then the group $K_{3}$ is a normal subgroup of index $6 p$ in $K_{0}$ that uniformizes a closed Riemann surface of genus $p$. In fact, for every integer $a$, we have $S^{a} \widehat{S}^{a} \in K_{3}$. Under the assumptions on $l$ and $k$, we have the equalities

$$
\begin{cases}U(S \widehat{S}) U^{-1} & =S\left(S^{k} A\right)^{-1}\left(S^{k-1}(S \widehat{S}) S^{-k+1}\right)\left(S^{-1}\left(S^{k} A\right) S\right) S^{-1} \\ V(S \widehat{S}) V & =\left(S^{l} B\right)^{-1}\left(S^{l-1}(S \widehat{S}) S^{-l+1}\right)\left(S^{l} B\right) \\ U\left(S^{k} A\right) U^{-1} & =S^{k}\left(S^{k} A\right)^{-1}\left(S^{k-1}(S \widehat{S}) S^{-k+1}\right)\left(S^{k-l-1}\left(S^{l} B\right) S^{-k+l+1}\right) S^{-k} \\ V\left(S^{k} A\right) V & =\left(S^{l} B\right)^{-1}\left(S^{-2 k}\left(S^{k} \widehat{S}^{k}\right)\left(S^{k} A\right)^{-1} S^{2 k}\right)\left(S^{l} B\right) \\ U\left(S^{l} B\right) U^{-1} & =S^{l}\left(S^{k} A\right)^{-1} S^{-l} \\ V\left(S^{l} B\right) V & =\left(S^{l} B\right)^{-1}\left(S^{l} \widehat{S}^{l}\right) .\end{cases}
$$


The above ensures the normality of $K_{3}$ in $K_{0}$. That $K_{3}$ has index $6 p$ in $K_{0}$ follows from the fact that a fundamental domain for $K_{3}$ is given by $P_{3}=P_{2} \cup$ $S\left(P_{2}\right) \cup \cdots \cup S^{-1}\left(P_{2}\right)$. The surface uniformized by $K_{3}$ admits the conformal automorphisms $S$ of order $p$ with quotient the orbifold uniformized by the group $K_{2}$. The rest is just consequence of Poincaré's polygon theorem.

Now, under the conditions on $k$ and $l$ as above, we have

$$
\begin{aligned}
K_{0} / K_{3} & =\left\langle u, v: u^{3 p}=v^{2}=(v u)^{6}=u^{3} v u^{3} v=u^{3 k-1} v u v=u^{3 l+1} v u^{-1} v=1\right\rangle \\
& =\left\langle u, v: u^{3 p}=v^{2}=1, v u v=u^{t p-1}\right\rangle,
\end{aligned}
$$

where $t \in\{0,1,2\}$. We claim that $t \neq 0$. In fact, $t=0$ will assert that $K_{0} / K_{3}$ is the dihedral group of order $6 p$, a contradiction to the fact that every dihedral conformal action on a closed Riemann surface is of Schottky type and, in particular, cannot quotient to an orbifold of genus zero with exactly three branch values $[6,7]$. If we denote by $(a, b)$ the greatest common divisor between $a$ and $b$, then we have the following. In the case $t=1$ we should have $(3, p-1)=1$. In fact, on the contrary we have $(3, p-1)=3$ and we obtain that the presentation of $K_{0} / K_{3}$ gives a group of order $2 p$. Similarly, in the case $t=2$ we should have $(3,2 p-1)=1$. As a consequence of all the above, we have obtained Corollary 3.

\section{References}

[1] Edge, W. L.: Bring's curve. J. London Math. Soc. (2) 18 (1978), no. 3, 539-545.

[2] Farkas, H, and Kra, I.: Riemann surfaces. Second edition. Graduate Texts in Mathematics 71. Springer-Verlag, New-York, 1992.

[3] GonzÁlez-Díez, G.: Loci of curves which are prime Galois coverings of $P^{1}$. Proc. London Math. Soc. (3) 62 (1991), 469-489.

[4] González-Díez, G. And Harvey, W. J.: Moduli of Riemann surfaces with symmetry. In Discrete groups and geometry, 75-93. London Math. Society Lecture Note Ser. 173. Cambridge University Press, Cambridge, 1992.

[5] González-Díez, G., Hidalgo, R. A. and Leyton, M.: Generalized Fermat's curves. Preprint.

[6] Hidalgo, R. A.: Dihedral groups are of Schottky type. Proyecciones 18 (1999), 23-48.

[7] Hidalgo, R. A.: On Schottky groups with automorphisms. Ann. Acad. Sci. Fenn. Ser. A I Math. 19 (1994), 259-289.

[8] Hidalgo, R. A.: Homology coverings of Riemann surfaces. Tôhoku Math. J. (2) 45 (1993), 499-503. 
[9] Hidalgo, R. A.: A commutator rigidity for function groups and Torelli's theorem. Proyecciones 22 (2003), 117-125.

[10] Hidalgo, R. A.: Noded function groups. In Complex geometry of groups (Olmué, 1998), 209-222. Contemp. Math., 240. Amer. Math. Soc., Providence, RI, 1999.

[11] Hidalgo, R. A.: Kleinian groups with common commutator subgroup. Complex Variables Theory Appl. 28 (1995), no. 2, 121-133.

[12] Keen, L.: Canonical polygons for finitely generated Fuchsian groups. Acta Math. 115 (1965), 1-16.

[13] Kuribayashi, A. And Kimura, H.: On automorphism groups of compact Riemann surfaces of genus 5. Proc. Japan Acad. Ser. A Math. Sci. 63 (1987), no. $4,126-130$.

[14] Kuribayashi, I. and Kuribayashi, A.: Automorphism groups of compact Riemann surfaces of genera three and four. J. Pure Appl. Algebra 65 (1990), no. 3, 277-292.

[15] Leyton, M.: Cubrimientos abelianos maximales. Masters Thesis presented to the Department of Mathematics, Universidad Técnica Federico Santa María, 2004.

[16] Macbeath, A. M.: On a curve of genus 7. Proc. London Math. Soc. (3) 15 (1965), 527-542.

[17] Maclachlan, C.: Abelian groups of automorphisms of compact Riemann surfaces. Proc. London Math. Soc. (3) 15 (1965), 699-712.

[18] Maskit, B.: Kleinian Groups. Grundlehren der Mathematischen Wissenschaften 287. Springer-Verlag, Berlin, 1988.

[19] Maskit, B.: The homology covering of a Riemann surface. Tôhoku Math. J. (2) 38 (1986), 561-562.

[20] NAG, S.: The complex analytic theory of Teichmüller spaces. Canadian Mathematical Society Series of Monographs and Advanced Texts. A WileyInterscience Publication. John Wiley \& Sons, New York, 1988.

[21] Rauch, H. E. and Lewittes, J.: The Riemann surface of Klein with 168 automorphisms. In Problems in analysis (papers dedicated to Salomon Bochner, 1969), 297-308. Princeton University Press, Princeton, New Jersey, 1970 .

[22] Riera, G. and Rodríguez, R.: The period matrix of Bring's curve. Pacific J. Math. 154 (1992), no. 1, 179-200.

[23] Ries, J.F.X.: Subvarieties of moduli space determined by finite group actions acting on surfaces. Trans. Amer. Math. Soc. 335 (1993), 385-406.

[24] Singerman, D.: Finitely maximal Fuchsian groups. J. London Math. Soc. (2) 6 (1972), 29-38. 
[25] Vermeulen, A. M.: Weierstrass points of weight two on curves of genus three. Dissertation, University of Amsterdam, Amsterdam, 1983. With a Dutch summary. Universiteit van Amsterdam, Amsterdam, 1983.

Recibido: 20 de abril de 2006

\author{
Maximiliano Leyton A. \\ Institut Fourier-UMR 5582 \\ Grenoble, France \\ leyton@fourier.ujf-grenoble.fr \\ Rubén A. Hidalgo \\ Departamento de Matemática \\ UTFSM Casilla 110-V \\ Valparaíso, Chile \\ ruben.hidalgo@usm.cl
}

Partially supported by Projects UTFSM 12.05.21 and Fondecyt 1070271. 